\title{
Impulsphotolytische Untersuchungen zum Mechanismus der photochemischen WoLfF-Umlagerung
}

\author{
Flash Light Photolytic Investigations Concerning \\ the Mechanism of the Photochemical WolfF Rearrangement \\ F. Bölsing und E. Spanuth* \\ Institut für organische Chemie der Technischen Universität Hannover
}

(Z. Naturforsch. 31 b, 1391-1396 [1976]; eingegangen am 31. Mai 1976)

\begin{abstract}
Wolff Rearrangement, Flash Light Photolysis, Kinetic Measurements, 9,10-Diazophenanthrone, Diphenylene Ketene
\end{abstract}

\begin{abstract}
9,10-Diazophenanthrone undergoes WoLfF rearrangement in alcoholic solvents and yields esters of 9-fluorene carboxylic acid. By flash light photolysis four intermediates could be detected. Kinetic measurements showed that the ketene formed by photolysis reacts with the solvent to give a cyclopentadienylic carbanion, which is protonated. A carbene or an oxirene intermediate could not be found.
\end{abstract}

Der Mechanismus der photochemischen WoLfFUmlagerung von $\alpha$-Diazocarbonylverbindungen ist Gegenstand einer Reihe von Arbeiten gewesen ${ }^{1}$. Von besonderem Interesse sind die im Reaktionsverlauf entstehenden Zwischenstufen, wobei vor allem die Frage nach der Beteiligung von Ketocarbenen und Oxirenen im Vordergrund steht.

Hinweise auf Ketocarbene ergeben sich u.a. aus Abfang- und Nebenreaktionen, wie die 1.3-dipolare Addition an $\mathrm{C}-\mathrm{C}$ - und $\mathrm{C}-\mathrm{N}$-Dreifachbindungen ${ }^{2,3}$, die Insertion, z. B. in O-H-Bindungen, oder die Reaktion mit schon gebildetem Keten zu DioxolDerivaten ${ }^{5}$. In demselben Sinne sind ESR-spektroskopische Untersuchungen an dem bei der Bestrahlung von Azibenzil bei $77^{\circ} \mathrm{K}$ in fester Matrix entstehenden Triplett-Ketocarben zu werten ${ }^{5-7}$.

Ein Nachweis der Oxiren-Zwischenstufe gelang mit Hilfe der Isotopenmarkierungstechnik (Photolyse von [Carbonyl-13C]-azibenzil). Die Experimente zeigen, daß Ketocarben und Oxiren in einer Gleichgewichtsbeziehung zueinander stehen ${ }^{8-10}$. Ferner weisen die bei der photochemischen Umsetzung spezieller arylsubstituierter Diazoketone erhaltenen Produkte auf die Beteiligung einer Oxiren-Zwischenstufe hin ${ }^{11}$.

* Teil der Dissertation von E. Spanuth, Technische Universität Hannover, 1976.

** Abb. 2 s. Tafel auf S. 1394a.

Sonderdruckanforderungen an Prof. Dr. F. BöLsING, Institut für organische Chemie der Technischen Universität, Schneiderberg 1B, D-3000 Hannover.
Im Zusammenhang mit Untersuchungen zur Photochromie von Diazoverbindungen ${ }^{12}$ haben wir versucht, die bei der Photolyse von $\alpha$-Diazocarbonylverbindungen diskutierten $Z$ wischenstufen unter den Reaktionsbedingungen der photochemischen WoLFF-Umlagerung durch Impulsphotolyse spektroskopisch nachzuweisen. Als Modellsubstanz wurde das 9.10-Diazophenanthron (1) gewählt.

\section{Ergebnisse und Diskussion}

Bei der Impulsphotolyse von $\mathbf{1}$ in Methanol, 2-Propanol und 2-Butanol lassen sich die Absorptionsbanden von insgesamt vier Zwischenstufen beobachten; die zugehörigen Absorptionsmaxima liegen bei 430, 490, 560 und 700/780 nm (Abbn. 1 und 2).

Bei den $Z$ wischenstufen mit $\lambda_{\max }=430$ und $700 /$ $780 \mathrm{~nm}$ (Z 430 und Z 700/780) erfolgt nach dem Anregungsblitz eine sofortige Abnahme der Extinktion. Im Gegensatz hierzu nimmt die Extinktion bei den Zwischenstufen mit $\lambda_{\max }=490$ und $560 \mathrm{~nm}$ (Z 490 und Z 560) zunächst zu, und erst nach Überschreiten eines Maximums erhält man das Bild einer normalen Abklingkurve (Abb. 2)**. Dementsprechend können nur $Z 430$ und $Z 700 / 780$ in einer photochemischen Primärreaktion entstanden sein, nicht aber Z 490 und Z 560, die offensichtlich in einer Dunkelreaktion aus einer vorgelagerten reaktiven Spezies gebildet werden. 


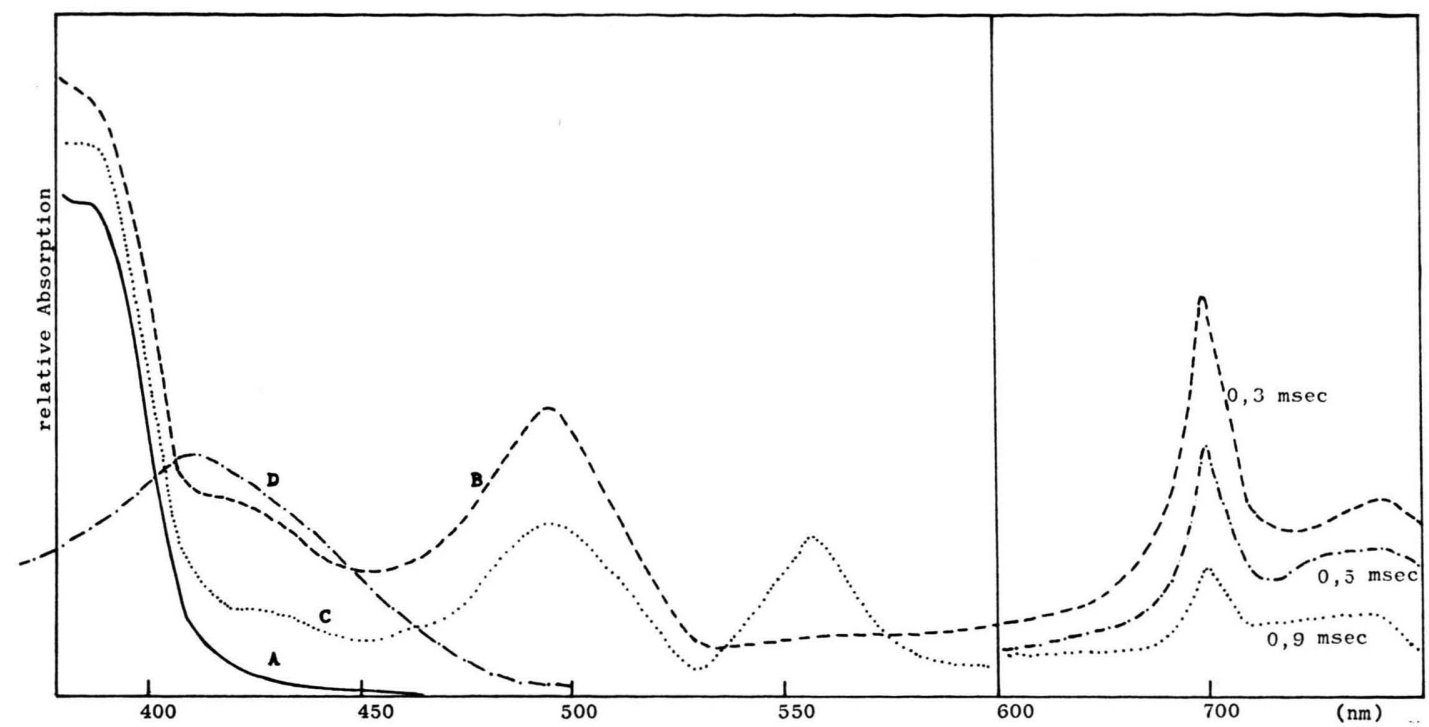

Abb. 1. Absorptionsspektren der bei der Impulsphotolyse von 9.10-Diazophenanthron auftretenden $\mathrm{Zwischen-}$ stufen. 1,7 $\times 10^{-4} \mathrm{Mol} \mathrm{l}^{-1}$ (2-Propanol):

A: Ausgangssubstanz,

B: 0,3 msec nach dem Blitz,
C: 1,5 msec nach dem Blitz,

D: zum Vergleich - Diphenylketen in Cyclohexan.
Z 430 ließ sich durch Vergleich mit einer authentischen Probe spektroskopisch als Diphenylenketen (2) identifizieren (Abb. 1). Z 700/780 ist im Gegensatz zu den übrigen Zwischenstufen in Gegenwart von Sauerstoff nicht faßbar. Dieser Befund deutet darauf hin, daß es sich um eine Spezies im TriplettZustand handelt.

Es liegt nahe, sowohl 2 als auch Z 700/780 als Vorstufen zu Z 490 bzw. Z 560 aufzufassen. Da die beiden letztgenannten $Z$ wischenstufen auch dann auftreten, wenn $Z$ 700/780 in Gegenwart von Sauerstoff nicht beobachtet wird, kommt gegebenenfalls nur noch 2 als Vorstufe in Frage. Da 2 in nucleophilen, protischen Lösungsmitteln zu den entsprechenden Carbonsäurederivaten abreagiert, besteht die Möglichkeit, daß Z 490 und Z 560 aus dem Keten im Zuge dieser Reaktion entstehen.

In diesem Zusammenhang ist es von Bedeutung, daß Z 560 offenbar nicht in einer Folgereaktion aus Z 490 gebildet wird. Für diesen Fall nämlich müßte die Steigung der Funktion $\mathrm{E}_{\mathrm{Z} 560}=\mathrm{f}(\mathrm{t})$ dort einen Maximalwert aufweisen, wo die entsprechende Extinktionskurve von Z490 ein Extionktionsmaximum besitzt; eine derartige Beziehung besteht jedoch nicht (Abb. 3).

Andererseits beobachtet man, daß die mittlere Lebensdauer von Z 560 wie auch die Intensität der Absorption mit steigender Basizität des Reaktions-

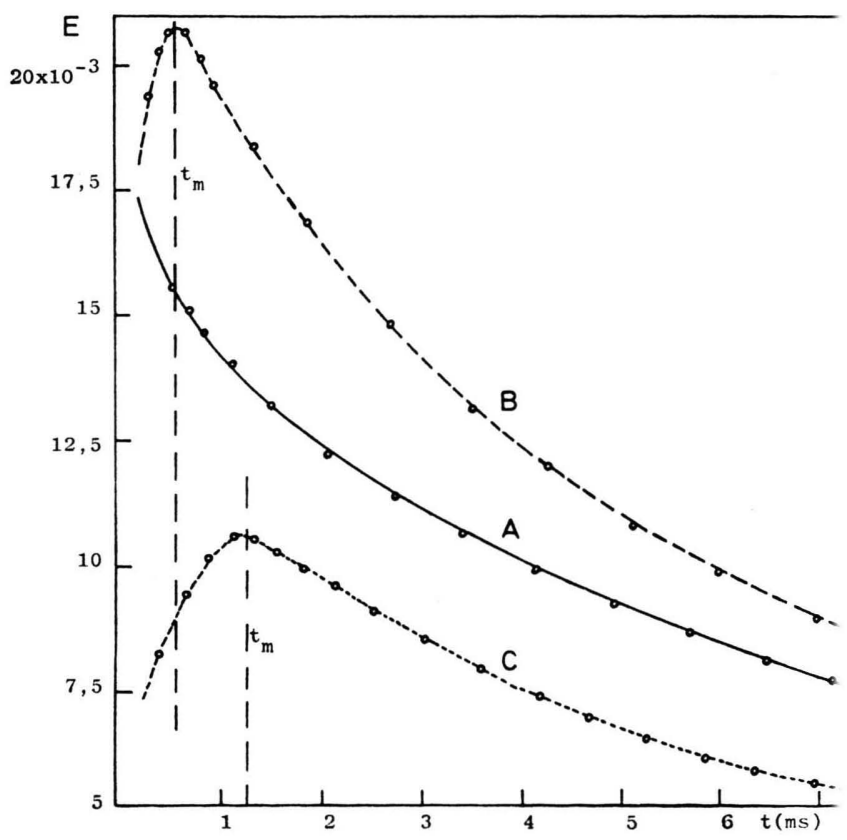

Abb. 3. Verlauf der absoluten Extinktionen von Z 430 (A), Z 490 (B) und Z 560 (C).

(Die Extinktionswerte von $\mathrm{Z} 560$ wurden um den Faktor 3 größer eingetragen.)

mediums zunimmt. Man kann deshalb davon ausgehen, daß im Verlaufe der Umsetzung des Diphenylenketens mit Alkohol zum 9-Fluorencarbonsäureester Z 560 als Zwischenstufe auftritt. 


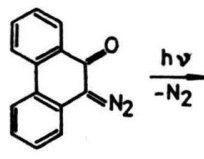

1

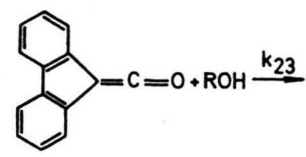

2

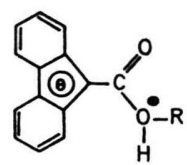

3

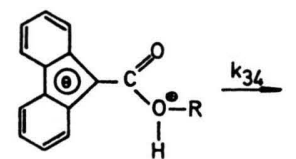

3

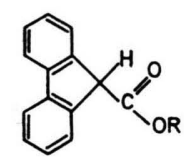

4
Die Reaktionen wurden in verdünnter alkoholischer Lösung durchgeführt. Dementsprechend sind die Reaktionsschritte 1. bzw. pseudo 1. Ordnung und die Rückreaktion $\mathbf{3} \rightarrow \mathbf{2}$ ist vernachlässigbar. Unter dieser Voraussetzung gelten die Differentialgleichungen

$$
\begin{aligned}
\frac{\mathrm{dc}_{2}}{\mathrm{dt}} & =-\mathrm{k}_{23} \mathrm{c}_{2} \\
\frac{\mathrm{dc}}{\mathrm{dt}} & =\mathrm{k}_{23} \mathrm{c}_{2}-\mathrm{k}_{34} \mathrm{c}_{3}
\end{aligned}
$$

Nach Einführung der gemessenen Extinktionsänderungen $\Delta \mathrm{E}$ und $\Delta \mathrm{E}_{\infty}$ gilt

$$
\begin{aligned}
& \left.\frac{\mathrm{dE}_{2}}{\mathrm{dt}}=-\mathrm{k}_{23} \mathrm{E}_{2) \mathrm{E}}=\Delta \mathrm{E}-\Delta \mathrm{E}_{\infty}\right) \\
& \frac{\mathrm{dE}_{3}}{\mathrm{dt}}=\frac{\varepsilon_{3}}{\varepsilon_{2}} \mathrm{k}_{23} \mathrm{E}_{2}-\mathrm{k}_{34} \mathrm{E}_{3}
\end{aligned}
$$

Durch die Integration von Gl. (3) erhält man

$$
\ln E_{2}=\ln E_{2}{ }^{0}-k_{23} \mathrm{t}
$$

Die Auftragung von $\operatorname{lnE}_{2}$ gegen $t$ muß demnach eine Gerade mit der Steigung $\mathbf{k}_{23}$ ergeben.
Die Werte $\mathrm{E}_{3}{ }^{\mathrm{m}}$ und $\mathrm{E}_{2}{ }^{\mathrm{m}}$ zum Zeitpunkt $\mathrm{t}^{\mathrm{m}}$ des Extinktionsmaximums von 3 sind aus der Messung bekannt (Abb. 3), so daß die Extinktionskoeffizienten $\varepsilon_{2}$ und $\varepsilon_{3}$ aus Gl. (4) eliminiert werden können. Zum Zeitpunkt $t_{m}$ gilt:

$$
\frac{\mathrm{dE}_{3}}{\mathrm{dt}}=\frac{\varepsilon_{3}}{\varepsilon_{2}} \mathrm{k}_{23} \mathrm{E}_{2} \mathrm{~m}-\mathrm{k}_{34} \mathrm{E}_{3} \mathrm{~m}=0
$$

Mit

$$
\frac{\varepsilon_{3}}{\varepsilon_{2}}=\frac{\mathrm{k}_{34}}{\mathrm{k}_{23}} \mathrm{Q}_{32^{\mathrm{m}}} ; \mathrm{Q}_{32^{\mathrm{m}}}=\mathrm{E}_{3}{ }^{\mathrm{m}} / \mathrm{E}_{2}{ }^{\mathrm{m}}
$$

erhält man aus Gl. (4)

$$
\frac{\mathrm{dE}_{3}}{\mathrm{dt}}=\mathrm{k}_{34}\left(\mathrm{E}_{2} \mathrm{Q}_{32}{ }^{\mathrm{m}}-\mathrm{E}_{3}\right)
$$

Die Geschwindigkeitskonstante $\mathrm{k}_{34}$ kann durch graphische Differentation der Funktion $E_{3}=f(t)$ mit Gl. (8) berechnet werden.

Integration von Gl. (2) und Einsetzen der gemessenen Extinktionswerte führt zu

$$
\mathrm{E}_{3}=\mathrm{E}_{2}{ }^{\circ} \mathrm{Q}_{32}{ }^{\mathrm{m}} \frac{\mathrm{k}_{34}}{\mathrm{k}_{34}-\mathrm{k}_{23}}\left(\mathrm{e}^{-\mathrm{k}_{23} \mathrm{t}}-\mathrm{e}^{-\mathrm{k}_{34} \mathrm{t}}\right)
$$

Sofern nun die mit Hilfe von Gl. (9) aus den gemessenen Geschwindigkeitskonstanten $\mathrm{k}_{23}$ und $\mathrm{k}_{34}$ berechneten Werte für $\mathrm{E}_{3}$ mit den zugehörigen gemessenen Extinktionswerten übereinstimmen, kann

\begin{tabular}{|c|c|c|c|c|c|c|c|}
\hline $\begin{array}{l}\mathrm{t} \\
{[\mathrm{ms}]}\end{array}$ & $10^{3} \cdot \mathrm{E}_{2}$ & $\ln E_{2}$ & $\begin{array}{l}10^{3} \cdot \mathrm{E}_{3} \\
\text { (gem.) }\end{array}$ & $\begin{array}{l}10^{3} \cdot \mathrm{E}_{3} \\
\text { (ber.) }\end{array}$ & $\begin{array}{l}10^{3} \\
\left(\mathrm{E}_{2} \mathrm{Q}_{32} \mathrm{~m}^{\mathrm{m}}-\mathrm{E}_{3}\right)\end{array}$ & $\mathrm{dE}_{3} / \mathrm{dt}$ & $\begin{array}{l}10^{3} \mathrm{k}_{34} \\
{\left[\mathrm{sec}^{-1}\right]}\end{array}$ \\
\hline 0,5 & 6,70 & $-5,005$ & 6,35 & 5,84 & 7,83 & 7,46 & 0,9527 \\
\hline 0,7 & 5,32 & $-5,236$ & 6,75 & 6,82 & 4,51 & $\mathbf{4 , 4 3}$ & 0,9823 \\
\hline 0,9 & 4,20 & $-5,473$ & 7,01 & 7,32 & 1,88 & 1,68 & 0,8936 \\
\hline 1,1 & 3,36 & $-5,696$ & 7,11 & 7,47 & 0 & 0 & \\
\hline 1,3 & 2,76 & $-5,892$ & 6,88 & 7,36 & $-1,04$ & $-\mathbf{I}, 00$ & 0,9615 \\
\hline 1,5 & 2,31 & $-6,070$ & 6,61 & 7,09 & $-1,72$ & $-1,70$ & 0,9884 \\
\hline 1,7 & 1,96 & $-6,234$ & 6,32 & 6,71 & $-2,17$ & $-2,08$ & 0,9585 \\
\hline 1,9 & 1,70 & $-6,376$ & 6,06 & 6,26 & $-2,46$ & $-2,35$ & 0,9550 \\
\hline 2,1 & 1,49 & $-6,509$ & 5,82 & 5,77 & $-2,67$ & $-2,55$ & 0,9553 \\
\hline 2,3 & 1,31 & $-6,638$ & 5,58 & 5,28 & $-2,81$ & $-2,67$ & 0,9502 \\
\hline 2,5 & 1,14 & $-6,776$ & 5,36 & 4,79 & $-2,95$ & $-2,80$ & 0,9492 \\
\hline 2,9 & 0,89 & $-7,024$ & 4,93 & 4,50 & $-3,05$ & $-2,84$ & 0,9311 \\
\hline 3,1 & 0,78 & $-7,156$ & 4,70 & 4,47 & $-3,05$ & $-2,84$ & 0,9311 \\
\hline 3,3 & 0,69 & $-7,279$ & 4,51 & 4,30 & $-3,05$ & $-2,83$ & 0,9279 \\
\hline
\end{tabular}
man davon ausgehen, daß der Reaktionsablauf den zugrunde gelegten Zeitgesetzen für Folgereaktionen 1.Ordnung entspricht. Es läßt sich so zeigen, daß der zunächst anhand qualitativer Befunde formulierte Mechanismus in der Tat zutrifft.

In Tab. I sind die gemessenen und berechneten Extinktionswerte der Reaktion in Methanol für

Tab. I. Kinetische Daten für die Reaktion in Methanol, Zeitbereich 0-3,3 msec ${ }^{18}$.

$\mathrm{k}_{23}=(0,86 \mathrm{I} \pm 0,188) \cdot 10^{3} \mathrm{sec}^{-1}, \mathrm{k}_{34}=(0,949 \pm 0,067) \cdot 10^{3} \mathrm{sec}^{-1}$. 


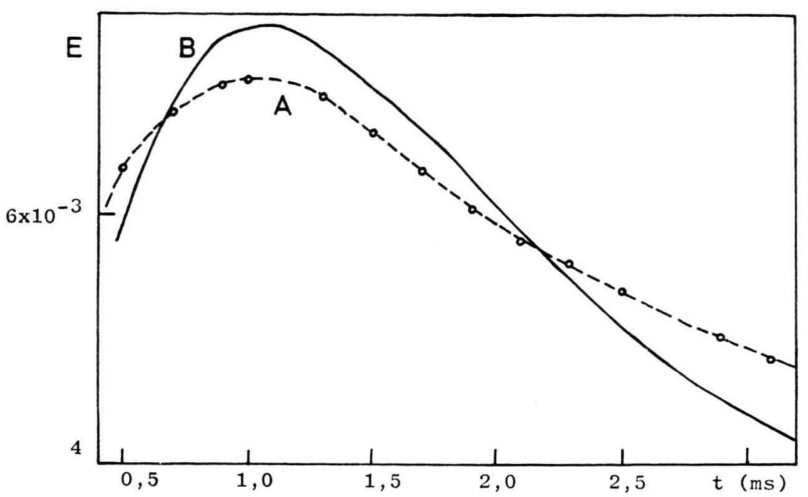

Abb. 4. Beobachteter und berechneter Verlauf der Extinktion von 3 in Methanol (0-3,3 ms). A: gemessen, B: berechnet.

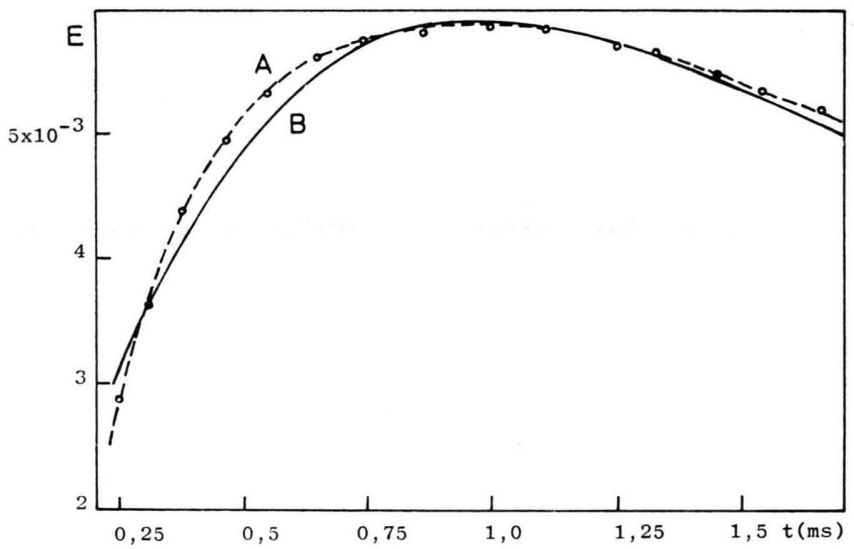

Abb. 5. Beobachteter und berechneter Verlauf der Extinktion von $\mathbf{3}$ bei der Reaktion in Methanol (0-1,7 ms).

A: gemessen,

B: berechnet. einen Zeitbereich bis zu 3,3 msec aufgeführt. Nach dieser Reaktionszeit hat ein Umsatz von $92 \%$ in bezug auf Diphenylenketen stattgefunden. Bei einer zweiten Messung (Tab. II und Abb.5) wurde der Zeitbereich gestreckt und der Reaktionsverlauf bis $\mathrm{zu}$ einer Zeit von 1,7 msec und einem Umsatz von $80 \%$ in bezug auf das Keten aufgenommen.

Die zweite Messung ergibt wegen der höheren Meßgenauigkeit die zuverlässigeren Werte.

Wie den Tabellen zu entnehmen ist, besteht tatsächlich eine gute Übereinstimmung zwischen gemessenen und berechneten Daten.

Bei der Reaktion in 2-Propanol ist die Geschwindigkeit der Abreaktion von 2 um den Faktor 6 kleiner als in Methanol. Ein Verlauf nach einem Zeitgesetz 1. Ordnung wird nur bis $\mathrm{zu}$ einem Umsatz von etwa $40 \%$ beobachtet; danach treten $\mathrm{Ab}$ weichungen auf (Tab. III).

Die Ursache hierfür kann in der Meßmethode selbst liegen ${ }^{13}$, zum anderen könnte $\mathbf{2}$ oder auch $\mathbf{3}$ mit einer weiteren Zwischenstufe, z.B. Z 490, in einem Gleichgewicht vorliegen. Die experimentellen Ergebnisse lassen eine eindeutige Erklärung nicht zu. Von diesen Abweichungen abgesehen, folgt jedoch auch dieses Reaktionssystem dem angenommenen Mechanismus. Der Zeitpunkt $\mathrm{t}_{\mathrm{m}}$ des Extinktionsmaximums von 3 ist gegenüber dem der Reaktion in Methanol zu längeren Zeiten verschoben; gleichzeitig hat die relative Konzentration zum Zeitpunkt $t_{m}$ in bezug auf das Keten abgenommen.

Beides steht im Einklang mit der Tatsache, daß $\mathrm{k}_{23}$ beim Übergang von Methanol nach 2-Propanol kleiner, $\mathrm{k}_{34}$ jedoch größer geworden ist. Allgemein

Tab. II. Kinetische Daten für die Reaktion in Methanol, Zeitbereich 0-1,671 msec ${ }^{18}$.

\begin{tabular}{|c|c|c|c|c|c|c|c|}
\hline $\begin{array}{l}\mathrm{t} \\
{[\mathrm{ms}]}\end{array}$ & $10^{3} \cdot \mathrm{E}_{2}$ & $\ln \mathrm{E}_{2}$ & $\begin{array}{l}10^{3} \mathrm{E}_{3} \\
\text { (gem.) }\end{array}$ & $\begin{array}{l}10^{3} \mathrm{E}_{3} \\
\text { (ber.) }\end{array}$ & $\begin{array}{l}10^{3} \\
\left(\mathrm{E}_{2} \mathrm{Q}_{32}{ }^{\mathrm{m}}-\mathrm{E}_{3}\right)\end{array}$ & $\mathrm{dE}_{3} / \mathrm{dt}$ & $\begin{array}{l}10^{3} \mathrm{k}_{34} \\
{\left[\mathrm{sec}^{-1}\right]}\end{array}$ \\
\hline 0,250 & 6,30 & $-5,067$ & 2,86 & 3,14 & 12,37 & 10,58 & 0,8553 \\
\hline 0,306 & 5,67 & $-5,172$ & 3,63 & 3,63 & 10,08 & 9,35 & 0,9276 \\
\hline 0,374 & 5,09 & $-5,280$ & 4,41 & 4,13 & 7,89 & 7,30 & 0,9252 \\
\hline 0,465 & 4,45 & $-5,414$ & 4,94 & 4,70 & 5,82 & 5,50 & 0,9450 \\
\hline 0,550 & 4,00 & $-5,521$ & 5,36 & 5,10 & 4,31 & 4,01 & 0,9304 \\
\hline 0,650 & 3,56 & $-5,637$ & 5,62 & 5,46 & 2,99 & 2,85 & 0,9532 \\
\hline 0,743 & 3,23 & $-5,735$ & 5,73 & 5,68 & 2,01 & 1,94 & 0,9652 \\
\hline 0,865 & 2,81 & $-5,874$ & 5,81 & 5,85 & 0,98 & 0,88 & 0,8970 \\
\hline 1,000 & 2,42 & $-6,024$ & 5,85 & 5,90 & 0 & 0 & \\
\hline 1,109 & 2,13 & $-6,151$ & 5,83 & 5,87 & $-0,68$ & $-0,65$ & 0,9558 \\
\hline 1,250 & 1,81 & $-6,314$ & 5,71 & 5,73 & $-1,34$ & $-1,19$ & 0,8881 \\
\hline 1,336 & 1,64 & $-6,413$ & 5,64 & 5,63 & $-1,68$ & $-1,54$ & 0,9166 \\
\hline 1,450 & 1,45 & $-6,536$ & 5,50 & 5,45 & $-2,00$ & $-1,88$ & 0,9400 \\
\hline 1,542 & 1,34 & $-6,615$ & 5,37 & 5,29 & $-2,13$ & $-1,86$ & 0,8732 \\
\hline 1,671 & 1,22 & $-6,708$ & 5,22 & 5,03 & $-2,27$ & $-2,05$ & 0,9031 \\
\hline
\end{tabular}

$\mathrm{k}_{23}=(1,097 \pm 0,005) \cdot 10^{3} \mathrm{sec}^{-1}, \mathrm{k}_{34}=(0,919 \pm 0,0087) \cdot 10^{3} \mathrm{sec}^{-1}$. 
F. Bölsing-E. Spanuth · Photochemische WolfF-Umlagerung

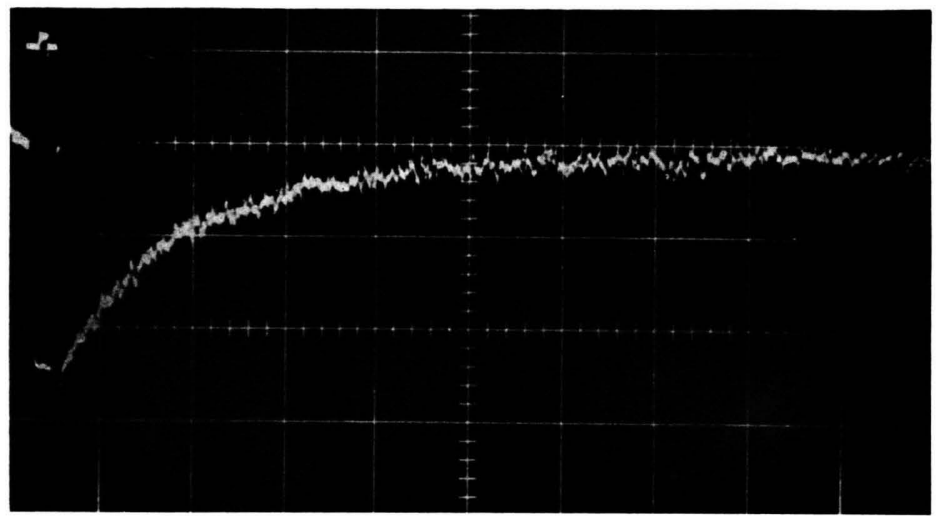

$\lambda=430 \mathrm{~nm}$,

$2,7 \times 10^{-4} \mathrm{Mol} \mathrm{l}^{-1}$ in Methanol,

Osz.-Empf. 0,05 V/cm,

Ablenkgeschw. 1,0 msec/cm.

A

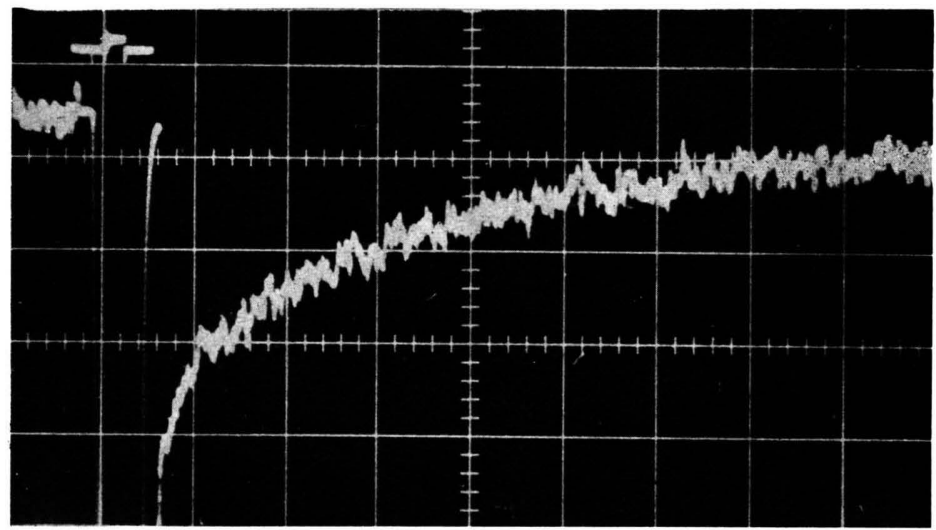

$\lambda=780 \mathrm{~nm}$,

$1,7 \times 10^{-4} \mathrm{Mol} \mathrm{l}^{-1}$ in 2-Propanol, Osz.-Empf. 0,01 V/cm,

Ablenkgeschw. 0,5 msec/cm.

B

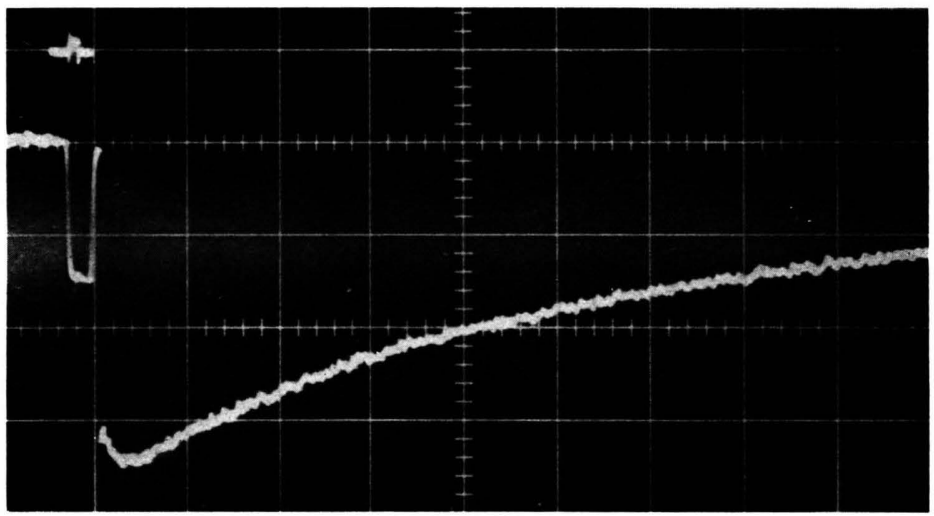

$\lambda=490 \mathrm{~nm}$

$1,7 \times 10^{-4} \mathrm{Mol} \mathrm{1}^{-1}$ in 2-Propanol,

Osz.-Empf. 0,05 V/cm,

Ablenkgeschw. 1,0 msec $/ \mathrm{cm}$.

C

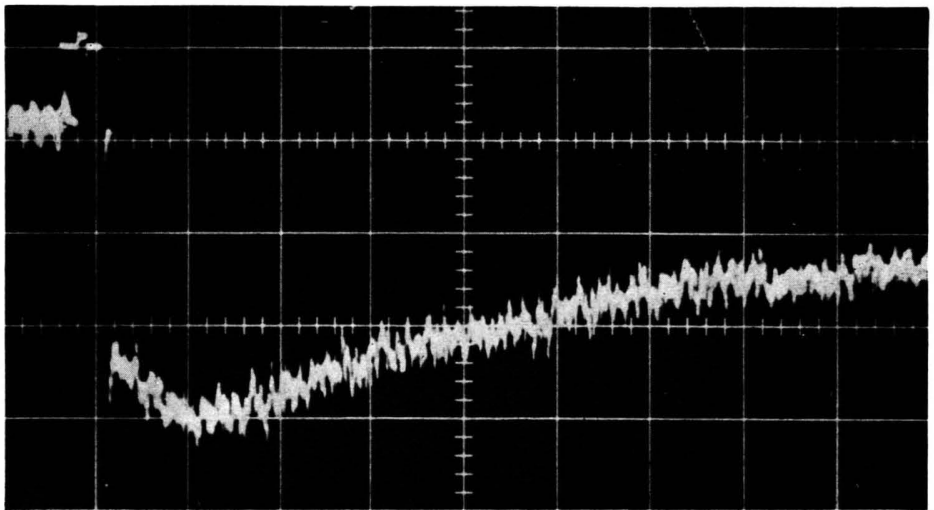

$\lambda=560 \mathrm{~nm}$

$1,7 \times 10^{-4} \mathrm{Mol} \mathrm{1}^{-1}$ in 2-Propanol, Osz.-Empf. 0,01 V/cm,

Ablenkgeschw. 1,0 msec/cm.

D

Abb. 2. Absorptionsverlauf der bei der Impulsphotolyse von 1 erfaßten Zwischenstufen.

Zeitschrift für Naturforschung 31 b, Seite 1394 a. 
Tab. III. Kinetische Daten für die Reaktion in 2-Propanol ${ }^{18}$.

\begin{tabular}{|c|c|c|c|c|c|c|c|}
\hline $\begin{array}{l}\mathrm{t} \\
{[\mathrm{ms}]}\end{array}$ & $10^{2} \cdot \mathrm{E}_{2}$ & $\begin{array}{l}10^{-2} \cdot \mathrm{k}_{23} \\
{\left[\mathrm{sec}^{-1}\right]}\end{array}$ & $\begin{array}{l}10^{3} \cdot \mathrm{E}_{3} \\
\text { (gem.) }\end{array}$ & $\begin{array}{l}10^{3} \cdot \mathrm{E}_{2} \\
\text { (ber.) }\end{array}$ & $\begin{array}{l}10^{3} \\
\left.\mathrm{E}_{2} \mathrm{Q}_{32} \mathrm{~m}-\mathrm{E}_{3}\right)\end{array}$ & $\mathrm{dE}_{3} / \mathrm{dt}$ & $\begin{array}{l}10^{-3} \cdot \mathrm{k}_{34} \\
{\left[\mathrm{sec}^{-1}\right]}\end{array}$ \\
\hline 0,387 & 2,594 & 1,368 & 2,660 & 1,937 & 1,230 & 2,17 & 1,764 \\
\hline 0,476 & 2,560 & 1,389 & 2,748 & 2,210 & 1,093 & 1,80 & 1,647 \\
\hline 0,589 & 2,512 & 1,444 & 2,910 & 2,492 & 0,858 & 1,55 & 1,807 \\
\hline 0,621 & 2,506 & 1,408 & 2,958 & 2,559 & 0,802 & 1,40 & 1,746 \\
\hline 0,767 & 2,448 & 1,445 & 3,105 & 2,816 & 0,568 & 0,95 & 1,673 \\
\hline 0,887 & 2,401 & 1,436 & 3,215 & 2,972 & 0,387 & 0,65 & 1,680 \\
\hline 0,928 & 2,391 & 1,448 & 3,250 & 3,015 & 0,337 & 0,55 & 1,632 \\
\hline 1,032 & 2,358 & 1,437 & 3,320 & 3,106 & 0,217 & 0,38 & 1,782 \\
\hline 1,178 & 2,316 & 1,412 & 3,378 & 3,193 & 0,097 & 0,16 & 1,650 \\
\hline 1,300 & 2,263 & 1,457 & 3,395 & 3,240 & 0 & 0 & 0 \\
\hline 1,427 & 2,232 & 1,424 & 3,380 & 3,267 & $-0,032$ & $-0,06$ & 1,875 \\
\hline 1,590 & 2,191 & 1,392 & 3,323 & 3,278 & $-0,035$ & $-0,06$ & 1,715 \\
\hline 1,960 & 2,090 & 1,372 & 3,183 & 3,232 & $-0,047$ & $-0,08$ & 1,702 \\
\hline 2,154 & 2,057 & 1,323 & 3,102 & 3,184 & $-0,016$ & $-0,25$ & - \\
\hline 3,597 & 1,823 & 1,128 & 2,600 & 2,675 & 0,135 & $-0,36$ & - \\
\hline 4,995 & 1,668 & 0,990 & 2,133 & 2,120 & 0,250 & $-0,19$ & - \\
\hline 6,544 & 1,516 & 0,902 & 1,837 & 1,767 & 0,437 & $-0,16$ & - \\
\hline 8,166 & 1,409 & 0,812 & 1,625 & 1,404 & 0,488 & $-0,10$ & - \\
\hline
\end{tabular}

$\mathrm{k}_{23}=(1,418 \pm 0,0082) \cdot 10^{2} \mathrm{sec}^{-1}, \mathrm{k}_{34}=(1,720 \pm 0,0209) \cdot 10^{3} \mathrm{sec}^{-1}$.

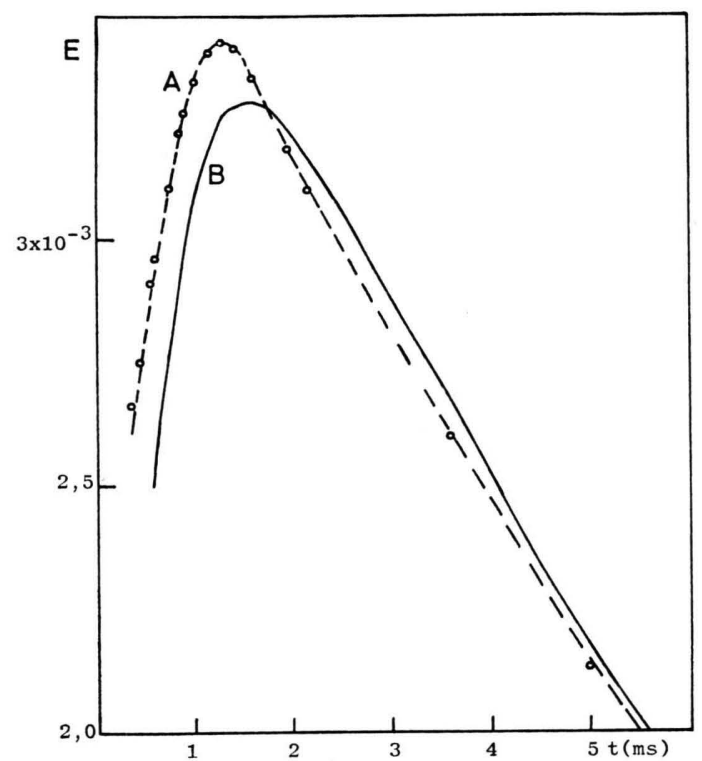

Abb. 6. Beobachteter und berechneter Verlauf der Extinktion von 3 bei der Reaktion in 2-Propanol.

A : gemessen,

B: berechnet.

führt bei Folgereaktionen eine schnellere Abreaktion der Zwischenstufe zu einer Verminderung der ,,stationären Konzentration", eine Verlangsamung seiner Bildung führt $\mathrm{zu}$ einer Verschiebung von $t_{m}$ nach höheren Werten. Beide Effekte treten hier auf. Eine Erhöhung von $\mathrm{k}_{34}$ in 2-Propanol zeigt zudem, daß die Lebensdauer von Z 560 solvationsabhängig ist. Dagegen scheint der nucleophile Angriff des
Alkoholmoleküls auf das Carbonyl-C-Atom des Ketens von den sterischen Gegebenheiten im Alkoholmolekül abhängig zu sein. Bei der Reaktion in 2-Butanol konnte bei $560 \mathrm{~nm}$ aufgrund dieser Effekte bereits keine Absorption mehr erfaßt werden, die Absorption bei $490 \mathrm{~nm}$ ist nach wie vor vorhanden.

Eine Zuordnung dieser Zwischenstufe ist nach den vorliegenden experimentellen Ergebnissen nicht möglich. Es kann jedoch ausgeschlossen werden, daß es sich dabei um ein Ketocarben handelt, da dieses in einem früher liegenden Zeitbereich des Reaktionsgeschehens auftreten müßte.

Tab. IV. Kinetische Daten für die Reaktion in 2-Butanol 18 .

\begin{tabular}{rlll}
\hline $\begin{array}{l}\mathrm{t} \\
{[\mathrm{msec}]}\end{array}$ & $10^{3} \cdot \mathrm{E}_{2}$ & $\ln \mathrm{E}_{2}$ & $\begin{array}{l}10^{-2} \mathrm{k}_{23} \\
{\left[\mathrm{sec}^{-1}\right]}\end{array}$ \\
\hline 1,11 & 4,463 & $-5,411$ & 0,70 \\
1,50 & 4,319 & $-5,444$ & 0,74 \\
2,18 & 4,110 & $-5,494$ & 0,73 \\
2,66 & 3,959 & $-5,531$ & 0,74 \\
3,27 & 3,749 & $-5,586$ & 0,77 \\
4,00 & 3,587 & $-5,630$ & 0,74 \\
4,69 & 3,421 & $-5,677$ & 0,73 \\
5,58 & 3,146 & $-5,761$ & 0,76 \\
6,59 & 2,985 & $-5,814$ & 0,72 \\
7,77 & 2,740 & $-5,899$ & 0,72 \\
8,67 & 2,612 & $-5,947$ & 0,70 \\
9,77 & 2,446 & $-6,013$ & 0,69 \\
10,70 & 2,317 & $-6,067$ & 0,68 \\
11,80 & 2,178 & $-6,129$ & 0,67 \\
12,80 & 2,042 & $-6,193$ & 0,67 \\
13,70 & 1,983 & $-6,223$ & 0,64 \\
14,70 & 1,966 & $-6,231$ & 0,61 \\
15,70 & 1,920 & $-6,255$ & 0,58 \\
16,70 & 1,901 & $-6,265$ & 0,55 \\
\hline
\end{tabular}

$\mathrm{k}_{23}=(0,72 \pm 0,0075) \cdot 10^{2}$. 
Tab. V. Gemessene Geschwindigkeitskonstanten der Reaktion Diphenylenketen und Alkohol ${ }^{18}$.

\begin{tabular}{llc}
\hline Lösungsmittel & $\mathrm{k}_{23}\left[\mathrm{sec}^{-1}\right]$ & $\mathrm{k}_{34}\left[\mathrm{sec}^{-1}\right]$ \\
\hline Methanol & $1,097 \times 10^{3}$ & $0,919 \times 10^{3}$ \\
Methanol & $0,861 \times 10^{3}$ & $0,949 \times 10^{3}$ \\
2-Propanol & $1,418 \times 10^{2}$ & $1,720 \times 10^{3}$ \\
2-Butanol & $0,720 \times 10^{2}$ & $\left(>1,72 \times 10^{3}\right)$ \\
\hline
\end{tabular}

\section{Experimentelles}

9.10-Diazophenanthron wurde nach der Vorschrift von CAVA, LitLe und NAPIER ${ }^{14}$ dargestellt, und nach Vorreinigung an $\mathrm{Al}_{2} \mathrm{O}_{3}$ in Methylenchlorid aus Cyclohexan/Petroläther umkristallisiert. Für die Darstellung des Diphenylenketen benutzten wir die Vorschrift von STAUDINGER ${ }^{15}$.

Die impulsphotolytischen Messungen wurden an einer Apparatur mit automatischer Kompensation des SEV-Ruhestromes ausgeführt16, 17. Die Anregung erfolgte durch zwei Blitzlampen der Fa. Heimann, Wiesbaden, vom Typ HG 702 (Länge $200 \mathrm{~mm}$;

1 L. Horner u. E. Spietschka, Chem. Ber. 85, 225 [1952]; Übersichtsartikel: H. MEIER u. K. P. ZELLER, Angew. Chem. 87, 52 [1975].

2 R. Huisgen, H. König, G. Binsch u. H. J. Sturm, Angew. Chem. 73, 368 [1961].

3 R. Huisgen, G. Binsch u. L. Ghozer, Chem. Ber. 97, 2628 [1964].

4 P. Yates u. E. W. Robb, J. Amer. Chem. Soc. 79, 5760 [1957].

5 A. M. Trozollo u. S. P. Fahrenholtz, Abstr. 151. Meeting Amer. Chem. Soc. 1966, K 23; A. M. Trozollo, Accounts Chem. Res. 1, 329 [1968].

${ }^{6}$ H. MeIER, IV. IUPAC Symp. Photochemistry 1972, 163.

7 Zum Einfluß der Spin-Multiplizität auf den Reaktionsablauf vgl.: A. PADWA u. R. Leyton, Tetrahedron Letters 1965, 2167; D. O. Cowan, M. M. Couch, K. R. Kopecky u. G. S. Hammond, J. Org. Chem. 29, 1922 [1964]; M. Jones (Jr.) u. W. Ander, J. Amer. Chem. Soc. 90, 2200 [1968].

8 K. P. Zeller, H. Meier, H. Kolshor u. E. Müller, Chem. Ber. 105, 1875 [1971].
$2,25 \mathrm{kV}$ je $10 \mu \mathrm{F})$. Die Meßlösung, die sich in einem 21 fassenden Vorratsgefä $\beta$ befand, wurde vor Beginn des Versuches mit gereinigtem Stickstoff von Sauerstoff befreit. Die Meßküvette (Länge $200 \mathrm{~mm}$, $\varnothing 20 \mathrm{~mm}$ ) ließ sich für jede Messung mit frischer Lösung aus dem Vorratsgefäß füllen, ohne daß diese mit der Atmosphäre in Berührung kommen konnte. Um eine Verfälschung der Meßdaten durch eventuell vom Anregungsblitz verursachtes Streulicht zu vermeiden, wurde der Eintrittsspalt des Monochromators für die Zeitdauer des Blitzes durch eine synchron gesteuerte Vorrichtung verschlossen. Zur Messung der Änderung des Photomultiplier-Stromes diente ein Oszillograph; die Oszillogramme wurden photographiert und die Negative vermessen.

Wir danken dem Institut für Biophysik der Technischen Universität Hannover, das uns freundlicherweise die Durchführung der impulsphotolytischen Messungen ermöglicht hat. Herrn Dr. M. KLENERT danken wir für seine besondere Unterstützung in diesem Zusammenhang.

9 D. E. Thornton, R. K. Gosavi u. O. P. Strausz, J. Amer. Chem. Soc. 92, 1768 [1970].

10 G. Frater u. O. P. Strausz, J. Amer. Chem. Soc. 92, 6654 [1970]

11 F. Bölsing u. K. NogaI, in Vorbereitung; K. NogaI, Dissertation, TU Hannover 1972.

12 F. Bölsing, Tetrahedron Letters 1968, 4299.

13 Die Ermittlung der absoluten Extinktion nach $\mathrm{E}=\Delta \mathrm{E}-\Delta \mathrm{E} \infty$ liefert keine richtigen Werte, wenn während der Reaktion eine stabile Spezies gebildet wird, deren Eigenabsorption im Absorptionsbereich der Zwischenstufe liegt.

14 M. P. Cava, R. L. Litle u. D. R. Napier, J. Amer. Chem. Soc. 80, 2262 [1958].

15 H. Staudinger, Ber. 29, 3062 [1906].

16 M. Klenert, Habilitationsschrift, Technische Universität Hannover 1976.

17 J. P. Keane u. C. Bell, Int. J. Phys. Chem. 5, 463 (Fig. 4) [1973].

18 Die Messungen wurden bei Raumtemperatur durchgeführt. 